Service social

Pratiques de conscientisation. Expériences d'éducation populaire au Québec, par G. Ampleman, G. Doré, L. Gaudreau, C. Larose, L. Leboeuf et D. Ventelou, Montréal, Nouvelle Optique, 1983, 304 pages.

\title{
Ronald Duhaime
}

Volume 34, numéro 2-3, 1985

L’organisation communautaire

URI : https://id.erudit.org/iderudit/706284ar

DOI : https://doi.org/10.7202/706284ar

Aller au sommaire du numéro

Éditeur(s)

École de service social de l'Université Laval

ISSN

1708-1734 (numérique)

Découvrir la revue

Citer ce compte rendu

Duhaime, R. (1985). Compte rendu de [Pratiques de conscientisation.

Expériences d'éducation populaire au Québec, par G. Ampleman, G. Doré, L.

Gaudreau, C. Larose, L. Leboeuf et D. Ventelou, Montréal, Nouvelle Optique, 1983, 304 pages.] Service social, 34(2-3), 410-411.

https://doi.org/10.7202/706284ar d'utilisation que vous pouvez consulter en ligne.

https://apropos.erudit.org/fr/usagers/politique-dutilisation/ 
L'ouvrage est rédigé dans un langage clair et accessible. La mise en page est agréable et agrémentée d'illustrations humoristiques. Une publication intéressante pour ceux et celles qui s'inscrivent dans ce modèle de pratique, en particulier dans le champ de la justice.

École de service social,

Gérald DORÉ

Université Laval.

Pratiques de conscientisation. Expériences d'éducation populaire au Québec, par G. Ampleman, G. Doré, L. Gaudreau, C. Larose, L. Lebofuf et D. Ventelou, Montréal, Nouvelle Optique, 1983, 304 pages.

Ce livre tant attendu des militantes et militants engagés dans la ligne de la conscientisation ne m'a pas déçu. II s'avère un outil précieux que j'utilise régulièrement dans ma pratique que je veux de plus en plus conscientisante.

Je fais d'abord une mise en garde. II ne faut pas y voir des recettes magiques, pas plus que chercher la théorie achevée de la conscientisation au Québec. Ce n'est pas un exposé abstrait et idéaliste de cette théorie.

II faut aborder ce livre pour ce qu'il est et pour ce que ses auteurs ont voulu qu'il soit, c'est-à-dire l'effort d'un collectif pour partager avec d'autres leurs expériences, leurs pratiques, les outils qu'ils et elles ont développés, et leur réflexion sur la conscientisation à partir de ce vécu. Ce n'est pas un bilan critique de leurs expériences.

L'ouvrage se divise en trois parties. La première présente des démarches de formation ou d'action où de multiples outils pédagogiques sont combinés dans un processus d'ensemble. On y trouve l'histoire de la lutte des assistés sociaux de Montréal sur la taxe d'eau; l'histoire de la Botte de foin, une maison de campagne collective; I'histoire du cheminement du journal de quartier Droit de parole. De plus, les auteurs décrivent deux sessions de formation : une sur la loi d'aide sociale et l'autre sur la "sensibilisation à la conscientisation" par les membres du Regroupement des organisateurs communautaires du Québec (R.O.C.Q.).

La deuxième partie est davantage consacrée à des outils spécifiques: l'image, l'écrit et le verbal; à la planification et l'évaluation; et le brassage des idées.

La troisième présente les acquis théoriques des auteurs. Cohérents avec la ligne de conscientisation qui part du vécu, qui est avant tout un processus inductif, ils ont d'abord voulu décrire leurs pratiques pour ensuite présenter leurs acquis théoriques. Cette troisième partie nous parle de l'origine et de l'évolution du concept et de la méthode de conscientisation chez Freire. Ensuite sont décrites les dimensions fondamentales de la conscientisation, les axes et les niveaux de conscience et les rôles et attitudes des intervenantes et des intervenants. 
Avec cet ouvrage, tout n'est pas dit sur les pratiques de conscientisation au Québec, au contraire. Ce que les auteurs nous communiquent, c'est l'état de leur cheminement au moment où le livre a été écrit.

Je termine en reprenant leurs paroles lors du lancement, à Québec : "Qui va écrire le prochain livre sur les pratiques de conscientisation au Québec?". J'espère que nous n'aurons pas trop longtemps à attendre.

\section{C.L.S.C. Sud-Ouest-Centre,}

Ronald DUHAIME Sherbrooke.

Guide méthodologique pour la recherche et l'action sociale, par André JACOB, Montréal, Nouvelle frontière, 1984, 65 pages.

Ce livre se situe d'emblée dans la catégorie des guides portant sur la méthodologie du travail intellectuel. Il vise en particulier les trois objectifs suivants: fournir les règles de base pour la recherche académique; renseigner sur les techniques de recherche et des sources d'information; outiller les étudiants et les étudiantes pour la réalisation de leurs travaux.

Cet ouvrage est divisé en sept parties. Dans la première, l'auteur distingue la recherche de la pratique sociale. Les suivantes traitent des critères pour une recherche progressiste; des types de recherche-action à privilégier; des premiers pas vers la recherche; comment réaliser la recherche; une conclusion générale; et une bibliographie, utile pour la recherche.

La recherche-action est habituellement entreprise dans le but de transformer une situation ou dans celui, plus modeste, d'énoncer des recommandations qui, mises en ouvre, changeraient cette situation. Selon que son but immédiat est de susciter des changements (par des processus aussi divers que l'innovation, l'animation sociale, le développement communautaire) ou d'étudier la situation pour éventuellement la transformer, les méthodologies peuvent varier considérablement (recherche, fonction mixte, animation sociale).

Rappelons que les options en recherche-action peuvent être résumées de la façon suivante: l'approche mécaniste; l'approche techno-systémique; l'approche organique; l'approche autogérée. Rappelons également que ces approches ne sont pas des étapes mais des visions particulières de la rechercheaction. C'est pourquoi nous ne pouvons comparer des conceptions différentes qui ne peuvent être placées sur une même ligne.

Dans le cas présent, l'auteur a choisi l'approche autogérée, qui se fonde sur les vues marxistes de la société. Les théoriciens du socialisme (Marx, Fourier, Proudhon) ont montré comment le mouvement ouvrier révélait, par ses luttes, les failles du système capitaliste. Le mouvement devenait alors l'analyseur du capitalisme industriel du 19 e siècle. D'autre part, l'aspiration profonde du socialisme se concrétisait dans une société autogérée qui devait suivre, en principe, la destruction de l'appareil d'état bureaucratique. 